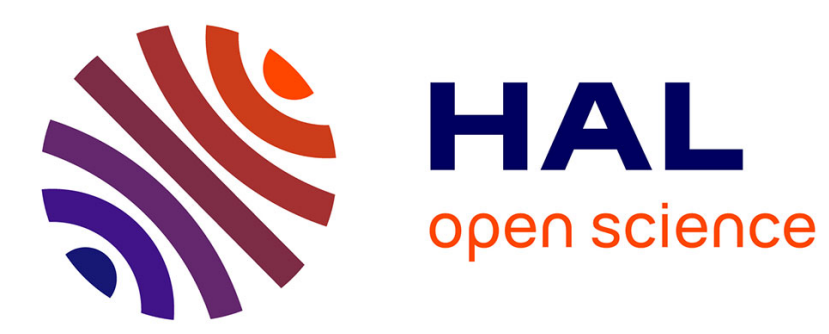

\title{
Global Identification of Joint Drive Gains and Dynamic Parameters of Parallel Robots
}

\author{
Sébastien Briot, Maxime Gautier
}

\section{To cite this version:}

Sébastien Briot, Maxime Gautier. Global Identification of Joint Drive Gains and Dynamic Parameters of Parallel Robots. Multibody System Dynamics, 2015, 33 (1). hal-00862322

\section{HAL Id: hal-00862322 \\ https://hal.science/hal-00862322}

Submitted on 25 Jun 2019

HAL is a multi-disciplinary open access archive for the deposit and dissemination of scientific research documents, whether they are published or not. The documents may come from teaching and research institutions in France or abroad, or from public or private research centers.
L'archive ouverte pluridisciplinaire HAL, est destinée au dépôt et à la diffusion de documents scientifiques de niveau recherche, publiés ou non, émanant des établissements d'enseignement et de recherche français ou étrangers, des laboratoires publics ou privés. 


\title{
Global Identification of Joint Drive Gains and Dynamic Parameters of Parallel Robots
}

\author{
Sébastien Briot • Maxime Gautier
}

Received: date / Accepted: date

\begin{abstract}
Off-line robot dynamic identification methods are based on the use of the Inverse Dynamic Identification Model (IDIM), which calculates the joint forces/torques (estimated as the product of the known control signal - the input reference of the motor current loop - with the joint drive gains) that are linear in relation to the dynamic parameters, and on the use of linear least squares technique to calculate the parameters (IDIM-LS technique). Most of the papers dealing with the dynamic parameters identification of parallel robots are based on simple models, which take only the dynamics of the moving platform into account. However, for advanced applications such as output force control in which the robot interaction force with the environment are estimated from the values of the input reference, both identifications of the full robot model and joint drive gains are required to obtain the best results. In this paper a systematic way to derive the full dynamic identification model of parallel robots is proposed in combination with a method that allows the identification of both robot inertial parameters and drive gains. The method is based on the total least squares solution of an over-determined linear system obtained with the inverse dynamic model. This model is calculated with available input reference of the motor current loop and joint position sampled data while the robot is tracking some reference trajectories without load on the robot and some trajectories with a known payload fixed on the robot. The method is experimentally validated on a prototype of parallel robot, the Orthoglide.
\end{abstract}

This work has been partially funded by the French ANR project ARROW (ANR 2011 BS3 $00601)$.

S. Briot

Institut de Recherche en Communications et Cybernétique de Nantes

IRCCyN, UMR CNRS 6597, Nantes, FRANCE

Tel.: +33(0)240376958

Fax: +33 (0)240376930

E-mail: Sebastien.Briot@irccyn.ec-nantes.fr

M. Gautier

LUNAM, Université de Nantes,

Institut de Recherche en Communications et Cybernétique de Nantes

IRCCyN, UMR CNRS 6597, Nantes, FRANCE 
Keywords Parallel robot · Force calibration - Drive gains · Inertial parameters identification · Total Least Squares

\section{Introduction}

Parallel robots have increasingly been used for a few decades. This is due to their main advantages compared to their serial counterparts that are: (i) a higher intrinsic rigidity, (ii) a larger payload-to-weight ratio and (iii) higher velocity and acceleration capacities [1]. In order to obtain these interesting properties, a good controller should be implemented. Several approaches could be envisaged [2,3], but it appears that, for high-speed robots or when varying loads have to be compensated for (e.g. in pick-and-place operations or machining), computed torque control is generally used [4]. This approach requires an accurate identification of the dynamic model of the robot with the load [5], which can be obtained if two main conditions are satisfied:

1. a well-tuned derivative band-pass filtering of actuated joints position is used to calculate the actuated joints velocities and accelerations, and

2. the values of actuator drive gains $g_{\tau}$ are accurately known to calculate the actuator force/torque as the product of the known control signal (computed by the numerical controller of the robot), i.e. the current references, by the drive gains.

However, it is often difficult or impossible to obtain robot manufacturers' data on joint drive gain values. And if available, data are usually given with an uncertainty greater than $10 \%$, thus leading to identification and force calibration errors. Thus, drive gains must be calibrated. Some methods have been developed in the past $[6,7]$ to calibrate the drive train constituted by a current controlled voltage source amplifier with gain $G_{i}$ which supplies a permanent magnet $D C$ or a brushless motor with torque constant $K_{t}$ coupled to the link directly or through gear train with gear ratio $N$. Each parameter was identified separately to then compute the drive gain $g_{\tau}=N G_{i} K_{t}$, but these procedures were very sensitive to measurement errors and time consuming, requiring heavy tests on the drive chain. This sensitivity to errors directly affects the accuracy of the output force estimation.

More recent works $[8,9]$ have proposed to identify the global joint drive gains $g_{\tau}$ for each actuated joint separately by using a known payload fixed on the endeffector. By carrying out with the robot two types of trajectories (trajectories with a known payload and trajectories without it), it appeared that it was possible to identify the payload terms using each joint $j$ equations. However, as the drive gain $g_{\tau_{j}}$ of joint $j$ is unknown, the estimated payload terms are indeed equal to the payload value divided by $g_{\tau_{j}}$. As the payload was known, it was thus possible to estimate $g_{\tau_{j}}$.

In [8], the approach requires the use of the ten payload inertial parameters estimated with $C A D$ software which implies some errors on the estimated values. Another drawback is to estimate the drive gain of one joint using only data coming from the corresponding joint equation which implies the loss of information about the coupled data on the other joints. As a result, we only succeed to identify the first four robot drive gains over six actuated joints. [9] is an improvement using only 
the accurate value of the weighed payload mass but with the drawback of using a sequential procedure for the computation of the payload inertial parameters that involves the accumulation of errors in the estimation of the drive gain parameters. Moreover, as previously, the estimation of the drive gain of one joint was done using only data coming from the corresponding joint equation which implies the loss of information about the coupled data on the other joints. With this approach, we only succeed in obtaining good results for the first four robot joints.

In this paper it is proposed a new method for the global identification of all robot dynamic parameters, including joint drive gains, using the input reference of the motor current loop and the joint position sampled data while the robot is tracking some reference trajectories without load fixed on the robot and some trajectories with a known payload fixed on the robot whose inertial parameters are measured or calculated with a $C A D$ software. Contrary to the previous works, all dynamic parameters and drive gains are calculated altogether as the total Least Square $(L S)$ solution of an over-determined system that takes into account the coupling between the robot axes.

This method is combined with the use of an Inverse Dynamic Identification Model $(I D I M)$ that takes into account all the robot link dynamic parameters. Indeed, most of papers dealing with dynamic parameters identification for parallel robots propose a simplified dynamic model that takes into account the platform and drive chain dynamics only [10-12]. Only few papers propose a systematic computation of the full IDIM. The most interesting and comprehensive works are presented in $[13,14]$, where the authors propose methods for computing the IDIM based on Jourdain's principle or Lagrange multipliers. The authors of [13] focus on the identification of the friction terms. They have proposed a way to decouple the identification procedure: first the inertial parameters are identified, then the friction terms. This decoupled identification is obtained through the use of special trajectories with constant velocities. This work however does not take into account the asymmetry in the friction coefficients. [14] is a pedagogical work on the identification of parallel robots which presents the identification procedure in detail. However, the method proposed to compute the base parameters may lead, for a parallel robot with identical legs (which is the case for most of parallel robots), to a set of base parameters which does not conserve the symmetry properties of the robot legs. Thus, the identification results are sometimes difficult to analyze. Furthermore, for all these works, the way to identify the drive gains is not treated and some Jacobian matrices, whose computation is not straightforward, are not clearly derived.

The present paper has two main goals:

- to propose a systematic and straightforward procedure for the computation of the IDIM for parallel robots which is combined with a way to obtain a set of symmetric base parameters when robot legs are identical,

- to present a new method for the global identification of all robot dynamic parameters, including joint drive gains.

The work will be decomposed as follows. First, in Section 2, the way to compute the IDIM for parallel robots is developed. A straightforward procedure is proposed for the computation of the Jacobian matrices required for closing the loops in the dynamic model. Section 3 presents the usual identification procedure. Moreover, some brief recalls are made for the computation of the base parameters of robots 
and the method is adapted to parallel robots so that, for a parallel robot with identical legs, it leads to a set of base parameters which conserves the symmetry properties of the robot legs. Then, Section 4 details the new procedure for the global identification of all robot dynamic parameters, including joint drive gains. In Section 5, the method is experimentally validated on a prototype of parallel robot developed at the IRCCyN: the Orthoglide [15].

A condensed version of this work has been presented in $[16,17]$. The present paper contains detailed proofs to enlighten the theoretical understanding of the method and gives additional experimental results to show its practical efficiency.

\section{Inverse Dynamic Identification Model of Parallel Robots}

\subsection{A Systematic Procedure for the IDIM Computation}

This work focuses only on parallel robots without actuation redundancy. A parallel robot is a complex multi-body system having several closed loops (Fig. 1(a)). It is composed of a moving platform connected to a fixed base by $n$ legs, each composed of $m_{i}$ elements. It is considered here that there is one actuator per leg, but the method can be easily extended to robots with several actuators per legs.

For the computation of IDIM of parallel robots, a method similar to the one presented in [18] is applied. The proposed method is decomposed into two steps:

1. first, all closed loops are virtually opened to make the platform virtually disassembled from the rest of the structure (Fig. 1(b)); each leg joint is virtually considered actuated (even for unactuated actual joints) so that the robot becomes a tree structure with a free body: the platform; the dynamic model of the tree structure and of the free platform is then computed using a systematic procedure based on the Newton-Euler principle,

2. then, the loops are closed using the loop-closure equations and the Lagrange multipliers, which involve the computation of robot Jacobian matrices.

In the following, the computation of the IDIM of the virtual tree structure and of the platform is recalled, and then a straightforward way to compute the Jacobian matrices for closing the loops is detailed.

\subsection{IDIM of Tree Open Loop Robots}

It is known that the complete rigid dynamic model of any open-loop tree structure can be linearly written in terms of a $\left(n_{t} \times 1\right)$ vector with respect to the standard parameters $\chi_{s t_{t}}$ [4] $\left(n_{t}\right.$ denotes the total number of joints for the virtual tree structure),

$$
\tau_{i d m_{t}}\left(\mathbf{q}_{t}, \dot{\mathbf{q}}_{t}, \ddot{\mathbf{q}}_{t}\right)=\mathbf{I D M}_{s t_{t}}\left(\mathbf{q}_{t}, \dot{\mathbf{q}}_{t}, \ddot{\mathbf{q}}_{t}\right) \chi_{s t_{t}}
$$

where $\tau_{i d m_{t}}$ is the $\left(n_{t} \times 1\right)$ vector of the input efforts of the virtual tree structure, $\mathbf{I D M}_{s t_{t}}$ is the $\left(n_{t} \times n_{s t_{t}}\right)$ Jacobian matrix of $\tau_{i d m_{t}}$, with respect to the $\left(n_{s t_{t}} \times 1\right)$ vector $\chi_{s t_{t}}$ of the standard parameters given by $\chi_{s t_{t}}^{T}=\left[\chi_{s t}^{1 T}, \chi_{s t}^{2 T}, \ldots \chi_{s t}^{n_{t} T}\right]$ and $\mathbf{q}_{t}, \dot{\mathbf{q}}_{t}, \ddot{\mathbf{q}}_{t}$ are the vectors of the joint positions, velocities and accelerations, respectively.

For rigid robots, the vector $\chi_{s t}^{j}$ of link $j$ is composed of 14 standard parameters described as: 


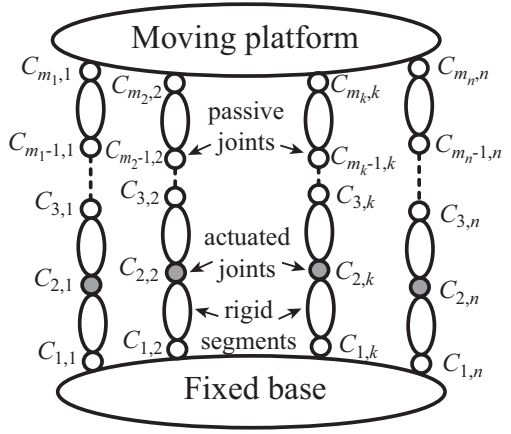

(a) Kinematic chain $\left(C_{j, k}\right.$ is the joint $j$ of the leg $k$, and $m_{k}$ is the total number of joints for the leg $k$ )

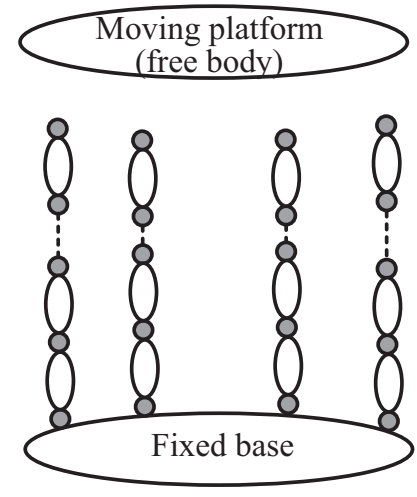

(b) Virtual tree structure

Fig. 1 A general parallel robot (the grey circles denote the actuated joints).

- $x x_{j}, x y_{j}, x z_{j}, y y_{j}, y z_{j}, z z_{j}$ are the 6 components of the inertia matrix of link $j$ at the origin of frame $j$,

- $m x_{j}, m y_{j}, m z_{j}$ are the 3 components of the first moment of link $j$,

- $m_{j}$ is its mass,

- $i a_{j}$ is the total inertia moment for rotor and gears of the drive train,

- $f v_{j}, f s_{j}$ are the viscous and Coulomb friction coefficients in the joint, respectively, and $\tau_{o f f_{j}}=\tau_{o f f f s_{j}}+\tau_{o f f \tau_{j}}$ is an offset parameter which regroups the current amplifier offset $\tau_{o f f \tau_{j}}$ and the asymmetrical Coulomb friction coefficient $\tau_{o f f f s_{j}}$.

In the same vein, the IDIM of the platform can be obtained as:

$$
\tau_{p}(\mathbf{x}, \mathbf{t}, \dot{\mathbf{t}})=\operatorname{IDM}_{p}(\mathbf{x}, \mathbf{t}, \dot{\mathbf{t}}) \chi_{p}
$$

where $\tau_{p}$ is the $(6 \times 1)$ vector of platform reaction wrench, $\mathbf{I D M}_{p}$ is the $(6 \times 10)$ Jacobian matrix of $\tau_{p}$, with respect to the $(10 \times 1)$ vector $\chi_{p}$ of the platform inertial standard parameters ${ }^{1}$ and $\mathbf{x}, \mathbf{t}, \dot{\mathbf{t}}$ are the platform position, twist and acceleration screw, respectively.

Several methods can be used to systematically derive these equations. Here, an algorithm based on the use of the modified Denavit-Hartenberg robot geometric description and the Newton-Euler principle is applied. This modeling is known to give the dynamic model equations in the most compact form [4].

\subsection{IDIM of Parallel Robots}

The IDIM of the virtual tree structure and of the free moving platform does not take into account the closed loop characteristics of parallel robots: among all joint

\footnotetext{
1 The number of standard parameters of a free rigid body can be reduced to 10 inertial parameters as it is not necessary to consider the parameters $i a_{j}, f v_{j}, f s_{j}$ and $\tau_{o f f_{j}}$ that are related to actuated joint drive chains.
} 
and platform coordinates $\mathbf{q}_{t}$ and $\mathbf{x}$ of the virtual robot (Fig. 1(b)), resp., only a subset denoted as $\mathbf{q}$ is independent in the real robot (the actual actuated joints positions, that are indeed a subset of $\mathbf{q}_{t}$ ). All these variables are linked through the loop-closure equations that can be obtained by expressing the (translational and rotational) displacement $\mathbf{x}_{k}$ of the last joints of each leg located at $C_{m_{k}, k}$ (that belong to both the platform and each leg $k$ - Fig. 1) in two different ways: (i) as a function of the platform coordinates $\mathbf{x}$ and (ii) as a function of all joint coordinates $\mathbf{q}_{t}$, such that:

$$
\mathbf{f}\left(\mathbf{x}, \mathbf{q}_{t}\right)=\left[\begin{array}{c}
\mathbf{x}_{1}(\mathbf{x})-\mathbf{x}_{1}\left(\mathbf{q}_{t}\right) \\
\vdots \\
\mathbf{x}_{n}(\mathbf{x})-\mathbf{x}_{n}\left(\mathbf{q}_{t}\right)
\end{array}\right]=\mathbf{0}
$$

The main problem with (3) is that, for most parallel robots, it is difficult to solve the forward kinematic problem $(f k p)$ using these equations in a straightforward way. Therefore, it is better to express the reduced loop-closure equations that directly relate the displacements $\mathbf{q}$ of the actuated joints to the moving platform coordinates $\mathbf{x}$ which is almost simpler to obtain [1]:

$$
\mathbf{f}_{p}(\mathbf{x}, \mathbf{q})=\mathbf{0}
$$

and to solve then the reduced $f k p$ that gives $\mathbf{x}$ as a function of $\mathbf{q}$. Obviously, this problem can be also tedious, but:

- the equations are simpler to solve than when using the equations (3),

- if the problem cannot be solved because the obtained polynomial equations have a too high degree, a numeric procedure can be used [1].

Then, once the values of $\mathbf{x}$ are found as a function of $\mathbf{q}$, it is possible to introduce them into (3) in order to express all joint coordinates $\mathbf{q}_{t}$ as a function of $\mathbf{x}$, and thus of $\mathbf{q}$. This problem is generally easy for usual parallel robots [1] and, even for more complicated cases, can now be solved using advanced mathematical methods [19].

Differentiating (3) and (4) with respect to time, the following expressions can be obtained:

$$
\begin{gathered}
\mathbf{A}_{p} \mathbf{v}+\mathbf{B}_{p} \dot{\mathbf{q}}=\mathbf{0} \Rightarrow \mathbf{v}=-\mathbf{A}_{p}^{-1} \mathbf{B}_{p} \dot{\mathbf{q}}=\mathbf{J}_{p} \dot{\mathbf{q}} \\
\mathbf{A}_{p} \dot{\mathbf{v}}+\dot{\mathbf{A}}_{p} \mathbf{v}+\mathbf{B}_{p} \ddot{\mathbf{q}}+\dot{\mathbf{B}}_{p} \dot{\mathbf{q}}=\mathbf{0} \\
\Rightarrow \dot{\mathbf{v}}=-\mathbf{A}_{p}^{-1}\left(\dot{\mathbf{A}}_{p} \mathbf{v}+\mathbf{B}_{p} \ddot{\mathbf{q}}+\dot{\mathbf{B}}_{p} \dot{\mathbf{q}}\right)
\end{gathered}
$$

and

$$
\begin{aligned}
& \mathbf{J}_{t k} \mathbf{v}-\mathbf{J}_{k} \dot{\mathbf{q}}_{t}=\mathbf{0} \Rightarrow \dot{\mathbf{q}}_{t}=\mathbf{J}_{k}^{-1} \mathbf{J}_{t k} \mathbf{v} \\
& \mathbf{J}_{t k} \dot{\mathbf{v}}+\dot{\mathbf{J}}_{t k} \mathbf{v}-\mathbf{J}_{k} \ddot{\mathbf{q}}_{t}-\dot{\mathbf{J}}_{k} \dot{\mathbf{q}}_{t}=\mathbf{0} \\
& \Rightarrow \ddot{\mathbf{q}}_{t}=\mathbf{J}_{k}^{-1}\left(\mathbf{J}_{t k} \dot{\mathbf{v}}+\dot{\mathbf{J}}_{t k} \mathbf{v}-\dot{\mathbf{J}}_{k} \dot{\mathbf{q}}_{t}\right)
\end{aligned}
$$

where

$$
\begin{aligned}
\mathbf{A}_{p} & =\left[\frac{\partial \mathbf{f}_{p}}{\partial \mathbf{x}_{i n d}}\right] \mathbf{T}, \mathbf{B}_{p}=\left[\frac{\partial \mathbf{f}_{p}}{\partial \mathbf{q}}\right] \\
\mathbf{J}_{t k} & =\left[\frac{\partial \mathbf{f}}{\partial \mathbf{x}_{i n d}}\right], \mathbf{J}_{k}=-\left[\frac{\partial \mathbf{f}}{\partial \mathbf{q}_{t}}\right]
\end{aligned}
$$


with $\mathbf{x}_{\text {ind }}$ a vector of the independent platform coordinates, that are a subset of $\mathbf{x}$, $\mathbf{T}$ a transformation matrix between the platform twist and the time derivatives of the terms $\mathbf{x}_{\text {ind }}$ [1], and $\mathbf{v}$ a vector of the independent coordinates in the platform twist $\left(\operatorname{dim} \mathbf{v}=\operatorname{dim} \mathbf{x}_{\text {ind }} \leq 6\right)$, i.e.

$$
\mathbf{t}=\mathbf{D} \mathbf{v}
$$

In the case of robots with 6 dof, $\mathbf{D}$ is the identity matrix. In these expressions, it is to be noted that

- the matrix $\mathbf{J}_{k}$ stacks all Jacobian matrices corresponding to the independent motions of the last joints of each leg due to the joint displacements of each serial leg and is thus a square matrix of dimension $\left(\left(n\left(m_{i}-1\right)\right) \times\left(n\left(m_{i}-1\right)\right)\right)$,

- the matrix $\mathbf{J}_{t k}$ is a matrix of dimension $\left(\left(n\left(m_{i}-1\right)\right) \times n\right)$ that can be obtained by considering the rigid body displacement of any point of the robot platform as a function of the platform twist, and

- in the case of parallel robots without actuation redundancy, the matrices $\mathbf{A}_{p}$ and $\mathbf{B}_{p}$ are square of dimension $(n \times n)$.

Finally, by introducing (5) and (6) into (7) and (8), the expressions of $\dot{\mathbf{q}}_{t}$ and $\ddot{\mathbf{q}}_{t}$ as a function of $\mathbf{q}, \dot{\mathbf{q}}$ and $\ddot{\mathbf{q}}$ can be obtained.

It should be mentioned that all the previous expressions are valuable as long as the robot does not meet any singularity and as long as the number of actuators is the same as the number of platform dof to control. Singularity avoidance or crossing is not the main topic of this paper, and the reader should refer to [20,21] for further developments. In the following of the Section 2.3, it is considered that all these matrices are regular.

To take into account the loop-closure constraints in the dynamic model of the parallel robot, Lagrange multipliers $\lambda^{T}=\left[\begin{array}{ll}\lambda_{1}^{T} & \lambda_{2}^{T}\end{array}\right]$ can be used [4] to compute the $(n \times 1)$ vector of the actuated joint force/torque $\tau_{i d m}$ of the closed-loop structure. $\tau_{i d m}$ can be obtained in relation of the Lagrange multipliers $\lambda$ by

$$
\tau_{i d m}=\left[\mathbf{0},-\mathbf{B}_{p}^{T}\right]\left[\begin{array}{c}
\lambda_{1} \\
\lambda_{2}
\end{array}\right]=-\mathbf{B}^{T} \lambda
$$

where $\lambda$ is calculated from the relation:

$$
\left[\begin{array}{cc}
\mathbf{J}_{k}^{T} & \mathbf{0} \\
-\mathbf{J}_{t k}^{T} & \mathbf{A}_{p}^{T}
\end{array}\right]\left[\begin{array}{c}
\lambda_{1} \\
\lambda_{2}
\end{array}\right]=\mathbf{A}^{T} \lambda=\left[\begin{array}{c}
\tau_{i d m_{t}} \\
\tau_{p r}
\end{array}\right]
$$

with $\mathbf{A}$ a square matrix of dimension $\left(\left(n m_{i}\right) \times\left(n m_{i}\right)\right)$ and

$$
\tau_{p r}=\mathbf{D}^{T} \tau_{p}
$$

where $\tau_{p}$ is defined in (2) and $\tau_{p r}$ is a subset of forces/moments in $\tau_{p}$ that can be found through the use of the principle of virtual powers, which states that:

$$
\mathbf{v}^{* T} \tau_{p r}=\mathbf{t}^{* T} \tau_{p}=\mathbf{v}^{* T} \mathbf{D}^{T} \tau_{p}
$$

In this equation, the superscript " "* stands for a virtual velocity.

It should also be mentioned that, in (11) and (12):

- $\lambda_{1}$ stacks the wrenches applied by the virtual tree structure on the platform at points $C_{m_{k}, k}$, 
$-\lambda_{2}$ stacks the values of the norms of the wrenches due to the platform dynamics in the platform joints located at $C_{m_{k}, k}$.

Thus, the second equation of the system (12) represents the platform equilibrium so that the loops of the parallel robots can be closed.

Solving (11) and using the right part of (5) and (7), it can be demonstrated that:

$$
\begin{aligned}
\tau_{i d m} & =\mathbf{J}_{t}^{T} \tau_{i d m_{t}}+\mathbf{J}_{p}^{T} \mathbf{D}^{T} \tau_{p} \\
& =\mathbf{J}_{t}^{T} \mathbf{I D M}_{s t_{t}} \chi_{s t_{t}}+\mathbf{J}_{p}^{T} \mathbf{D}^{T} \mathbf{I D M}_{p} \chi_{p} \\
& =\left[\mathbf{J}_{t}^{T} \mathbf{I D M}_{s t_{t}} \mathbf{J}_{p}^{T} \mathbf{D}^{T} \mathbf{I D M}_{p}\right]\left[\begin{array}{ll}
\chi_{s t_{t}}^{T} & \chi_{p}^{T}
\end{array}\right]^{T} \\
& =\mathbf{I D M}_{s t}(\mathbf{q}, \dot{\mathbf{q}}, \ddot{\mathbf{q}}) \chi_{s t}
\end{aligned}
$$

where $\mathbf{J}_{t}=\mathbf{J}_{k}^{-1} \mathbf{J}_{t k} \mathbf{J}_{p}$.

Finally, because of perturbations due to noise measurement and modeling errors, the actual force/torque $\tau$ differs from $\tau_{i d m}$ by an error e, such that:

$$
\tau=\tau_{i d m}+\mathbf{e}=\mathbf{I D M}_{s t} \chi_{s t}+\mathbf{e}
$$

where $\tau$ is calculated with the drive chain relation:

$$
\tau=\mathbf{v}_{\tau} \mathbf{g}_{\tau}=\left[\begin{array}{ccc}
v_{\tau_{1}} & 0 & 0 \\
0 & \ddots & 0 \\
0 & 0 & v_{\tau_{n}}
\end{array}\right]\left[\begin{array}{c}
g_{\tau_{1}} \\
\vdots \\
g_{\tau_{n}}
\end{array}\right]
$$

$\mathbf{v}_{\tau}$ is the $(n \times n)$ matrix of the actual motor current references of the current amplifiers $\left(v_{\tau_{j}}\right.$ corresponds to actuator $\left.j\right)$ and $\mathbf{g}_{\tau}$ is the $(n \times 1)$ vector of the joint drive gains $\left(g_{\tau_{j}}\right.$ corresponds to actuator $j$ ) that is given a priori by the manufacturer's data or measured with special time-consuming and heavy tests $[6$, 7]. Equation (16) represents the Inverse Dynamic Identification Model (IDIM).

\subsection{IDIM of Parallel Robots Including the Payload}

The payload is considered as an additional link (denoted as link $l$ ) fixed to the robot platform [5]. Only $n_{k l}$ among its ten parameters are considered to be known. The model (15) becomes:

$$
\tau_{i d m}=\left[\begin{array}{lll}
\mathbf{I D M}_{s t} & \mathbf{I D M}_{u l} & \mathbf{I D M}_{k l}
\end{array}\right]\left[\begin{array}{c}
\chi_{s t} \\
\chi_{u l} \\
\chi_{k l}
\end{array}\right]
$$

where:

$-\chi_{k l}$ is the $\left(n_{k l} \times 1\right)$ vector of the known inertial parameters of the payload;

$-\chi_{u l}$ is the $\left(n_{u l} \times 1\right)$ vector of the unknown inertial parameters of the payload, where $n_{u l}=10-n_{k l}$

- $\mathbf{I D M}_{k l}$ is the $\left(n \times n_{k l}\right)$ Jacobian matrix of $\tau_{i d m}$, with respect to the vector $\chi_{k l}$,

- IDM $_{u l}$ is the $\left(n \times n_{u l}\right)$ Jacobian matrix of $\tau_{i d m}$, with respect to the vector $\chi_{u l}$. 


\section{Usual Identification Procedure}

This part proposes a procedure for the computation of the base parameters for parallel robots so that, for a parallel robot with identical legs, it leads to a set of base parameters which conserves the symmetry properties of the robot legs, and then presents some necessary recalls on the identification procedure.

\subsection{Computation of the Base Parameters}

The off-line identification of the dynamic parameters is considered, given measured or estimated off-line data for $\tau$ and $(\mathbf{q}, \dot{\mathbf{q}}, \ddot{\mathbf{q}})$, collected while the robot is tracking some planned trajectories. The model (16) is sampled at frequency $f_{m}$ in order to get an over-determined linear system of $r_{f_{m}}$ equations and $n_{s t}$ unknowns:

$$
\mathbf{Y}_{f_{m}}(\tau)=\mathbf{W}_{f_{m}}^{s t}(\hat{\mathbf{q}}, \hat{\dot{\mathbf{q}}}, \hat{\ddot{\mathbf{q}}}) \chi_{s t}+\rho_{f_{m}}
$$

where $(\hat{\mathbf{q}}, \hat{\dot{\mathbf{q}}}, \hat{\ddot{\mathbf{q}}})$ is an estimation of $(\mathbf{q}, \dot{\mathbf{q}}, \ddot{\mathbf{q}})$, respectively, obtained by sampling and band-pass filtering the measure of $\mathbf{q}[22], \rho_{f_{m}}$ is the $\left(r_{f_{m}} \times 1\right)$ vector of errors, $\mathbf{Y}_{f_{m}}$ is the $\left(r_{f_{m}} \times 1\right)$ vector of the input torques/force, sampled at frequency $f_{m}$ and $\mathbf{W}_{f_{m}}^{s t}(\hat{\mathbf{q}}, \hat{\dot{\mathbf{q}}}, \hat{\mathbf{q}})$ is the $\left(r_{f_{m}} \times n_{s t}\right)$ observation matrix.

The force/torque $\tau$ is perturbed by high frequency unmodelled friction and flexibility force/torque of the joint drive chain which is rejected by the closed loop control. These force/torque ripples are eliminated with a parallel decimation procedure which low pass filters in parallel $\mathbf{Y}_{f_{m}}$ and each column of $\mathbf{W}_{f_{m}}^{s t}$ and resamples them at a lower rate, keeping one sample over $n_{d}$. This parallel decimation can be carried out with the MATLAB decimate function, where the low pass filter cut-off frequency, $\omega_{f p}=2 \pi 0.8 f_{m} /\left(2 n_{d}\right)$, is chosen in order to keep $\mathbf{Y}_{f_{m}}$ and $\mathbf{W}_{f_{m}}^{s t}$ in the same frequency range of the model dynamics. After the data acquisition procedure and the parallel decimation of (19), we obtain the over-determined linear system

$$
\mathbf{Y}(\tau)=\mathbf{W}^{s t}(\hat{\mathbf{q}}, \hat{\dot{\mathbf{q}}}, \hat{\ddot{\mathbf{q}}}) \chi_{s t}+\rho
$$

where $\rho$ is the $(r \times 1)$ vector of errors, $\mathbf{Y}$ is the $(r \times 1)$ vector of the input torques/force and $\mathbf{W}^{s t}(\hat{\mathbf{q}}, \hat{\dot{\mathbf{q}}}, \hat{\mathbf{q}})$ is the $\left(r \times n_{s t}\right)$ observation matrix.

In $\mathbf{Y}$ and $\mathbf{W}^{\text {st }}$, the equations of each joint $j$ are sorted in order to regroup the equations of each joint altogether such that: $\mathbf{Y}^{T}=\left[\left(\mathbf{Y}^{1}\right)^{T}, \cdots,\left(\mathbf{Y}^{n}\right)^{T}\right], \mathbf{W}^{s t}=$ $\left[\left(\mathbf{W}^{1}\right)^{T}, \cdots,\left(\mathbf{W}^{n}\right)^{T}\right]^{T}$, where $\mathbf{Y}^{j}$ and $\mathbf{W}^{j}$ represent the $r / n$ equations of joint $j$. It is to be noted that no error is introduced by the parallel filtering process in the linear relation (20) compared with (19). In [22], practical rules for tuning this filter are given.

The identifiable parameters are the base parameters which are the minimum number of dynamic parameters from which the dynamic model can be calculated [4]. The minimal dynamic model can be written using the $n_{b}$ base dynamic parameters $\chi$ as follows:

$$
\mathbf{Y}=\mathbf{W}(\mathbf{q}, \dot{\mathbf{q}}, \ddot{\mathbf{q}}) \chi+\rho
$$

where $\mathbf{W}$ is a subset of independent columns in $\mathbf{W}^{\text {st }}$ which defines the identifiable parameters. Several methods exist for the computation of these subsets (analytical [4] or numerical [23]). In this work, it is preferred to use a numerical method based on $Q R$ factorization. 
There is an infinity of possible subsets of base parameters, as presented in [14, 23]. In [14], the authors test different subsets (obtained via the $S V D$ of the observation matrix [23]) and keep the one which leads to the best conditioning index of the observation matrix. Even if it is computationally efficient, this method has a drawback: for a parallel robot with identical legs, it can lead to a set of base parameters which does not conserve the symmetry properties of the robot legs. Obviously, for avoiding this problem, it can be set a priori that some parameters are equivalent, which involves to sum their corresponding columns in the observation matrix. However, as there can be some small variations in the parameters values due to the manufacturing process, it is worth to avoid this a priori regrouping and check it a posteriori on the identified values.

Here, a method that avoids these drawbacks is described. For presenting it, let us make some brief recalls on the computation of the base parameters via $Q R$ factorization.

The $Q R$ factorization of the matrix $\mathbf{W}^{s t}$ of $(20)$ takes the form:

$$
\mathbf{Q}^{T} \mathbf{W}^{s t}=\left[\begin{array}{c}
\mathbf{R} \\
\mathbf{0}
\end{array}\right]
$$

where $\mathbf{Q}$ is a $r \times r$ orthogonal matrix and $\mathbf{R}$ is upper triangular.

If the absolute value $\left|R_{k k}\right|$ of the $k$-th component located on the diagonal of $\mathbf{R}$ is inferior to $\alpha$ ( $\alpha$ is the numerical rank - different from 0 because of round-off errors - and can be chosen such that $\alpha=\epsilon \max \left|R_{j j}\right|$, where $\epsilon$ is a small coefficient depending on the level of perturbations in $\mathbf{W}^{\text {st }}$ (due to noise measurement and error modelling) and $\max \left|R_{j j}\right|$ is the largest diagonal absolute value of $\mathbf{R}$ [23]), the $k$-th column $\mathbf{W}_{k}^{s t}$ of $\mathbf{W}^{s t}$ can be deleted. At the end of the procedure, $\left(n_{s t}-\right.$ $\left.n_{b}\right)$ columns of $\mathbf{W}^{s t}$ have been deleted that corresponds to $\left(n_{s t}-n_{b}\right)$ standard parameters removed from vector $\chi_{s t}$ to keep a set of $n_{b}$ base parameters $\chi$.

Because the $Q R$ algorithm starts from the last columns to the first of $\mathbf{W}^{s t}$, the $\left(n_{s t}-n_{b}\right)$ standard parameters to delete are dependent on the ordering of the columns of that matrix. For serial robots, the matrix $\mathbf{W}^{s t}$ is build such that the columns with the smaller indices are those corresponding to the links closest from the base. Thus, using the previous algorithm, the parameters with the smallest influence (those of the wrist) are eliminated from the base parameters.

For parallel robots, to take into account the symmetry in the leg dynamic parameters, it is preferable to order the columns of $\mathbf{W}^{s t}$ such that:

$$
\mathbf{W}_{r}^{s t}=\left[\begin{array}{llll}
\mathbf{W}_{p}^{s t} \mathbf{W}_{\chi_{1}, 1: n}^{s t} & \mathbf{W}_{\chi_{2}, 1: n}^{s t} \cdots & \mathbf{W}_{\chi_{n_{\text {st }}, g}, 1: n}^{s t}
\end{array}\right]
$$

where $n_{s t_{l e g}}$ is the number of standard parameters for one leg, matrix $\mathbf{W}_{p}^{s t}$ is the observation matrix corresponding to the platform inertial parameters and matrices $\mathbf{W}_{\chi_{k}, 1: n}^{s t}$ concatenates the columns of matrix $\mathbf{W}^{s t}$ corresponding to the parameters $\chi_{k}$ that are a priori identical for the $n$ legs. Then, $\left(n_{s t}-n_{b}\right)$ columns of $\mathbf{W}_{r}^{s t}$ can be deleted using the previous approach based on the $Q R$ factorization to obtain a new observation matrix $\mathbf{W}$ associated with a set of symmetrical base parameters denoted as $\chi$. 
3.2 Recalls on Least Squares Identification of the Base Dynamic Parameters $(I D I M-L S)$

Using the base parameters and tracking "exciting" reference trajectories, i.e. optimized trajectories that can be computed by nonlinear minimization of a criterion function of the condition number of the $\mathbf{W}^{s t}$ matrix [24,25], a well-conditioned matrix $\mathbf{W}^{\text {st }}$ can be obtained. Examples of such trajectories will be shown in Section 5 . The $L S$ solution $\hat{\chi}$ of (21) is given by:

$$
\hat{\chi}=\mathbf{W}^{+} \mathbf{Y}, \text { where } \mathbf{W}^{+}=\left(\mathbf{W}^{T} \mathbf{W}\right)^{-1} \mathbf{W}^{T}
$$

It is computed using the $Q R$ factorization of $\mathbf{W}$.

Standard deviations $\sigma_{\hat{\chi}_{i}}$ can be estimated assuming that $\mathbf{W}$ is a deterministic matrix and $\rho$ is a zero mean additive independent noise [22], with a covariance matrix $\mathbf{C}_{\rho \rho}$ such that

$$
\mathbf{C}_{\rho \rho}=E\left(\rho \rho^{T}\right)=\sigma_{\rho}^{2} \mathbf{I}_{r}
$$

$E$ is the expectation operator and $\mathbf{I}_{r}$, the $r \times r$ identity matrix. An unbiased estimation of the standard deviation $\sigma_{\rho}$ is:

$$
\sigma_{\rho}^{2}=\|\mathbf{Y}-\mathbf{W} \hat{\chi}\|^{2} /\left(r-n_{b}\right)
$$

The covariance matrix of the estimation error is given by:

$$
\mathbf{C}_{\hat{\chi} \hat{\chi}}=E\left[(\chi-\hat{\chi})(\chi-\hat{\chi})^{T}\right]=\sigma_{\rho}^{2}\left(\mathbf{W}^{T} \mathbf{W}\right)^{-1}
$$

$\sigma_{\hat{\chi}_{i}}^{2}=\mathbf{C}_{\hat{\chi} \hat{\chi}}(i, i)$ is the $i$-th diagonal coefficient of $\mathbf{C}_{\hat{\chi} \hat{\chi}}(27)$.

The ordinary $L S$ can be improved by taking into account different standard deviations on actuated joint $j$ equations errors [22]. Data in $\mathbf{Y}$ and $\mathbf{W}$ of (20) are weighted with the inverse of the standard deviation of the error calculated from ordinary $L S$ solution of the equations of joint $j[22]$

$$
\mathbf{Y}^{j}=\mathbf{W}^{j} \chi+\rho^{j}
$$

This weighting operation normalizes the errors in (20) and gives the weighted $L S$ estimation of the parameters (IDIM-WLS).

\subsection{Payload Identification}

In order to identify the dynamic parameters of both the robot and the payload, using the model (18), it is necessary that the robot carries out two types of trajectories $[26]$ :

1. trajectories without the payload, and

2. trajectories with the payload fixed to the end-effector.

The sampling and filtering of the model IDIM (18) can be then written as:

$$
\mathbf{Y}=\left[\begin{array}{ccc}
\mathbf{W}_{a} & \mathbf{0} & \mathbf{0} \\
\mathbf{W}_{b} & \mathbf{W}_{u l} & \mathbf{W}_{k l}
\end{array}\right]\left[\begin{array}{c}
\chi \\
\chi_{u l} \\
\chi_{k l}
\end{array}\right]+\rho
$$

where 
$-\mathbf{W}_{a}$ is the observation matrix of the robot in the unloaded case,

$-\mathbf{W}_{b}$ is the observation matrix of the robot in the loaded case,

- $\mathbf{W}_{u l}$ is the observation matrix of the robot corresponding to the unknown payload inertial parameters,

- $\mathbf{W}_{k l}$ is the observation matrix of the robot corresponding to the known payload inertial parameters.

Thus, these two types of trajectories avoid the regrouping of the payload parameters with those of the platform and allow their independent identification.

The next section presents the way to identify both link inertial parameters and joint drive gains.

\section{Global Identification of the Robot Dynamic Parameters and the Drive Gains}

In the usual IDIM-LS or IDIM-WLS, accurate values of the drive gains are necessary to compute vector $\mathbf{Y}$. However, it is often difficult or impossible to obtain manufacturers' data. And if available, data are given with an uncertainty greater than $10 \%$, thus leading to identification and force calibration errors. Therefore, it is preferable to introduce the drive gains into the base parameters and to use the Total Least Squares Identification (IDIM-TLS) procedure.

Details on the $T L S$ identification method can be found in [27] and many papers of the same authors. This method has been applied in [28] for the identification of the drive gains and the dynamic parameters on a two dof serial robot but gave arguable results due to the lack of an accurate scale factor.

In this paper a major improvement is proposed: the scaling of parameters using the accurate value of an additional payload mass.

4.1 Total Least Square Identification of the Robot Dynamic Parameters and the Drive Gain (IDIM-TLS)

Introducing (17) into (18), and keeping only the base parameters, we obtain:

$$
\tau=\mathbf{v}_{\tau} \mathbf{g}_{\tau}=\left[\operatorname{IDM}_{\mathbf{I D M}_{u l}} \mathbf{I D M}_{k l}\right]\left[\begin{array}{c}
\chi \\
\chi_{u l} \\
\chi_{k l}
\end{array}\right]+\mathbf{e}
$$

where IDM is the Jacobian matrix that relates the base parameters $\chi$ to the input torques $\tau$.

Then, using the method presented in the Section 3.3, the sampling and parallel decimation of the model IDIM (30) can be written as:

$$
\mathbf{Y}=\left[\begin{array}{c}
\mathbf{V}_{\tau a} \\
\mathbf{V}_{\tau b}
\end{array}\right] \mathbf{g}_{\tau}=\left[\begin{array}{ccc}
\mathbf{W}_{a} & \mathbf{0} & \mathbf{0} \\
\mathbf{W}_{b} & \mathbf{W}_{u l} & \mathbf{W}_{k l}
\end{array}\right]\left[\begin{array}{c}
\chi \\
\chi_{u l} \\
\chi_{k l}
\end{array}\right]+\rho
$$


where $\mathbf{V}_{\tau a}$ is the matrix of $\mathbf{v}_{\tau}$ samples in the unloaded case, $\mathbf{V}_{\tau b}$ is the matrix of $\mathbf{v}_{\tau}$ samples in the loaded case.

$$
\mathbf{V}_{\tau i}=\left[\begin{array}{ccc}
\mathbf{V}_{\tau i}^{1} & \cdots & \mathbf{0} \\
\vdots & \ddots & \vdots \\
\mathbf{0} & \cdots & \mathbf{V}_{\tau i}^{n}
\end{array}\right], \mathbf{V}_{\tau i}^{j}=\left[\begin{array}{c}
v_{\tau i, 1}^{j} \\
\vdots \\
v_{\tau i, r / n}^{j}
\end{array}\right] \text {, with } i=a, b
$$

in which $v_{\tau i, k}^{j}$ is the $k$-th sample of current reference for actuator $j, \mathbf{V}_{\tau i}^{j}$ regroups all the samples of the current reference for actuator $j$.

Rearranging the terms inside, Eq. (31) becomes:

$$
\mathbf{W}_{t o t} \chi_{t o t}=\left[\begin{array}{cccc}
-\mathbf{W}_{a} & \mathbf{V}_{\tau a} & \mathbf{0} & \mathbf{0} \\
-\mathbf{W}_{b} & \mathbf{V}_{\tau b} & -\mathbf{W}_{u l} & -\mathbf{W}_{k l} \chi_{k l}
\end{array}\right]\left[\begin{array}{c}
\chi \\
\mathbf{g}_{\tau} \\
\chi_{u l} \\
1
\end{array}\right]=\rho
$$

where $\mathbf{W}_{\text {tot }}$ is a $(r \times c)$ matrix $\left(c=\left(n_{b}+n+n_{u l}+1\right)\right)$ and $\chi_{\text {tot }}$ is a $(c \times 1)$ vector.

Without perturbation, $\rho=0$ and $\mathbf{W}_{\text {tot }}$ should be rank deficient to get the solutions $\lambda \chi_{\text {tot }} \neq \mathbf{0}$ depending on a scale coefficient $\lambda$. However because of the measurement perturbations, $\mathbf{W}_{\text {tot }}$ is a full rank matrix. Therefore, the system (33) is changed to the compatible system closest to (33):

$$
\hat{\mathbf{W}}_{t o t} \hat{\chi}_{t o t}=\mathbf{0}
$$

where $\hat{\chi}_{\text {tot }}^{T}=\left[\begin{array}{llll}\hat{\chi}^{T} & \hat{\mathbf{g}}_{\tau}^{T} & \hat{\chi}_{u l}^{T} & 1\end{array}\right]$ and $\hat{\mathbf{W}}_{\text {tot }}$ is the rank deficient matrix, closest to $\mathbf{W}_{\text {tot }}$ with respect to the Frobenius norm, i.e. $\mathbf{W}_{\text {tot }}$ minimizes the Frobenius norm $\left\|\mathbf{W}_{t o t}-\hat{\mathbf{W}}_{\text {tot }}\right\|_{F}[28] . \hat{\chi}_{t o t}$ is the solution of the compatible system (34) closest to $(33)$.

$\hat{\mathbf{W}}_{\text {tot }}$ can be computed thanks to the "economy size" $S V D$ of $\mathbf{W}_{\text {tot }}[29]$ :

$$
\mathbf{W}_{\text {tot }}=\mathbf{U}\left[\begin{array}{c}
\operatorname{diag}\left(s_{i}\right) \\
\mathbf{0}
\end{array}\right] \mathbf{V}^{T}
$$

where $\mathbf{U}$ and $\mathbf{V}$ are $(r \times r)$ and $(c \times c)$ orthonormal matrices, respectively, and $\operatorname{diag}\left(s_{i}\right)$ is a $(c \times c)$ diagonal matrix with singular values $s_{i}$ of $\mathbf{W}_{\text {tot }}$ sorted in decreasing order. $\hat{\mathbf{W}}_{\text {tot }}$ is given by:

$$
\hat{\mathbf{W}}_{\text {tot }}=\mathbf{W}_{t o t}-s_{c} \mathbf{U}_{c} \mathbf{V}_{c}^{T}
$$

where $s_{c}$ is the smallest singular value of $\mathbf{W}_{\text {tot }}$ and $\mathbf{U}_{c}\left(\mathbf{V}_{c}\right.$, resp.) the column of $\mathbf{U}$ (V, resp.) corresponding to $s_{c}$. Then, the normalized optimal solution $\hat{\chi}_{t o t}^{n}$ $\left(\left\|\hat{\chi}_{t o t}^{n}\right\|=1\right)$ is given by the last column of $\mathbf{V}, \hat{\chi}_{t o t}^{n}=\mathbf{V}_{c}[28]$.

There is infinity of vectors $\hat{\chi}_{t o t}=\lambda \hat{\chi}_{t o t}^{n}$ which are solution of (34) depending on a scale factor $\lambda$. The unique solution $\hat{\chi}_{t o t}^{*}=\hat{\lambda} \hat{\chi}_{t o t}^{n}$ for the robot can be found by taking into account that the last value $\hat{\chi}_{t^{*} t_{c}}^{*}$ of $\hat{\chi}_{\text {tot }}^{*}$ must be equal to 1 according to (33), i.e. $\hat{\lambda}=1 / \hat{\chi}_{t^{\prime} t_{c}}^{n}$, with $\hat{\chi}_{\text {tot }_{c}}^{n}$ the last value of $\hat{\chi}_{t o t}^{n}$. 
4.2 Statistical Analysis

Standard deviations $\sigma_{\hat{\chi}_{i}}$ on the dynamic and sensor gains parameters, are estimated assuming that all errors in data matrix $\mathbf{W}_{\text {tot }}$ are independently and identically distributed with zero mean and common covariance matrix $\mathbf{C}_{W W}$ such that

$$
\mathbf{C}_{W W}=\hat{\sigma}_{W}^{2} \mathbf{I}_{r_{W}}
$$

where $\mathbf{I}_{r_{W}}$ is the identity matrix of dimension $(r \times c) \times(r \times c)$.

An unbiased estimation of the standard deviation $\hat{\sigma}_{W}[27]$

$$
\hat{\sigma}_{W}=s_{c} / \sqrt{r-c}
$$

The covariance matrix of the estimation error is approximated by [27]:

$$
\mathbf{C}_{\hat{\chi} \hat{\chi}} \approx \hat{\sigma}_{W}^{2}\left(1+\left\|\hat{\chi}_{1: c-1}\right\|_{2}^{2}\right)\left(\hat{\mathbf{W}}_{\text {tot }_{1: c-1}}^{T} \hat{\mathbf{W}}_{t_{\text {ot }} t_{1: c-1}}\right)^{-1}
$$

with $\hat{\chi}_{1: c-1}$ the vector containing the $(c-1)$ first coefficients of $\hat{\chi}_{t o t}^{*}$ and $\hat{\mathbf{W}}_{t o t_{1: c-1}}$ a matrix composed of the $(c-1)$ first columns of $\hat{\mathbf{W}}_{t o t}$. Finally, $\sigma_{\hat{\chi}_{i}}^{2}=\mathbf{C}_{\hat{\chi} \hat{\chi}}(i, i)$ is the $i$-th diagonal coefficient of $\mathbf{C}_{\hat{\chi} \hat{\chi}}$ and the relative standard deviation $\% \sigma_{\hat{\chi}_{r i}}$ is given by: $\% \sigma_{\hat{\chi}_{r i}}=100 \sigma_{\hat{\chi}_{i}} /\left|\hat{\chi}_{i}\right|$, for $\left|\hat{\chi}_{i}\right| \neq 0$.

In order to improve the estimation of $\hat{\chi}_{t o t}^{*}$, the rows of $\mathbf{W}_{\text {tot }}$ are weighted taking into account the confidence on the measurements. As proposed in IDIM$W L S$ (Section 3.2), to improve the TLS solution, each row corresponding to joint $j$ equation is weighted by the inverse of $\hat{\sigma}_{W_{j}}$, i.e. the standard deviation corresponding to the data of the joint $j$ equations. Moreover, to take into account that the confidence on data in $\mathbf{V}_{\tau a, b}$ is higher than for data in $\mathbf{W}_{a, b}$ and $\mathbf{W}_{u, k l}$, the columns of $\mathbf{V}_{\tau a, b}$ could also be weighted. However, our experiments have shown that the results were not really improved; therefore this last weighting procedure was not used in the next section.

\subsection{Discussion on the A Priori Knowledge of the Payload Parameters}

The accuracy of $\hat{\lambda}$ depends on the accuracy of the knowledge $\chi_{k l}$ of the payload parameters. The parameters can be obtained by measurements or estimated by $C A D$ software. However, due to the possible errors in $C A D$ values, the most accurate payload parameter is the mass value $m_{l}$ that can be accurately measured using a weighing machine. For the robot under study in the next section, the Orthoglide, only the payload mass appears in the model (the other payload parameters have no effects on the dynamics). However, for other types of robots such as serial six dof robots, our experiments have shown that, due to the error on the parameter estimation using $C A D$ software, it is better to carry out the identification IDIMTLS using the knowledge of the payload mass only [30]. These results are not shown here as they are out of the scope of the paper. 


\section{Case Study: the Orthoglide Robot}

5.1 Description of the Architecture

The Orthoglide is a parallel robot with three translational dof composed of three identical legs (Fig. 2). Each leg is made of one linearly actuated foot linked at its extremity to a spatial parallelogram (Fig. 3(a)). The parallelogram is also attached to the mobile platform.

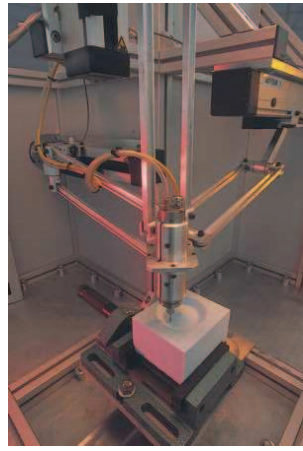

(a) Prototype

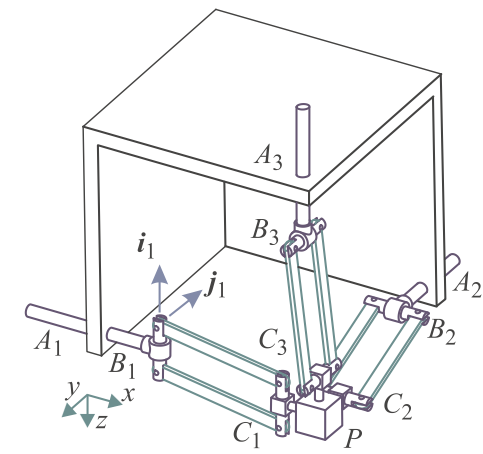

(b) Kinematic chain

Fig. 2 The Orthoglide robot.

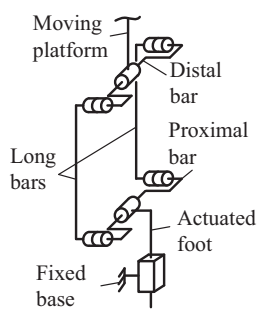

(a) Kinematics of one leg

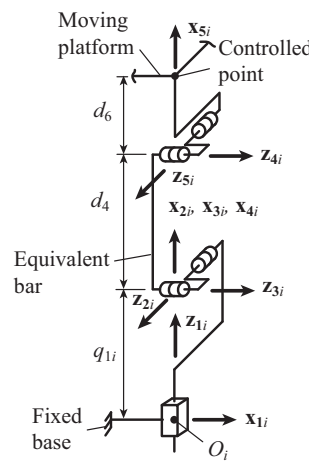

(b) Equivalent kinematics of one leg

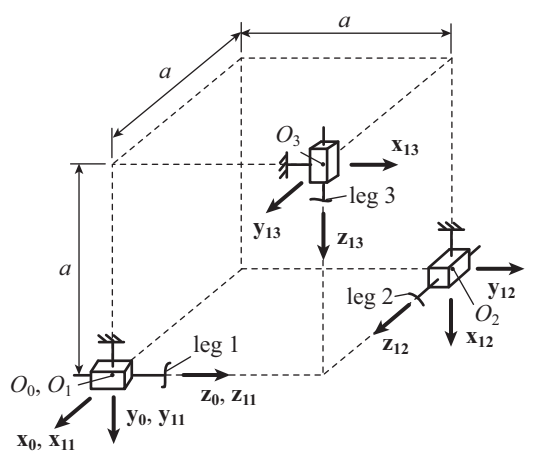

(c) Fixed base

Fig. 3 Kinematic chain description.

The directions of the three linear actuators of the Orthoglide are orthogonal (Fig. 2(b)). This aims at creating a mechanism with a workspace shape close to a cube and whose behavior is close to the isotropy wherever it is located in its workspace [15]. 
For the remainder of the paper, it should be mentioned that the gravity field $\mathbf{g}$ is directed along the vector $\mathbf{y}_{0}$ of Fig. 3(c).

For the dynamic modeling of the Orthoglide, as the bars of the parallelograms are parallel and as the prototype has been designed so that they are identical, it is possible to replace the long bars by only one equivalent bar linked at each extremity by two orthogonal pivots (Fig. 3(b)) without loss of generality. Using the previous assumption, the Orthoglide kinematics necessary for computing the dynamic model are defined using the modified Denavit and Hartenberg notation $(M D H)$ [4]. The geometric parameters of the virtual open-loop tree structure are given in Tables 1 and 2. The platform and payload are considered as supplementary bodies, the payload being fixed on the platform. They are respectively numbered as bodies 6 and 7 .

The $M D H$ notation being well known, the parameters of Tables 1 and 2 will not be defined here. For more information concerning the $M D H$ parameters, the reader should refer to [4].

Table 1 MDH parameters for the frames corresponding to the first body of each leg.

\begin{tabular}{|r|r|r|r|r|r|r|r|r|r|}
\hline$j_{i}$ & $a\left(j_{i}\right)$ & $\mu_{j i}$ & $\sigma_{j i}$ & $\gamma_{j i}$ & $b_{j i}$ & $\alpha_{j i}$ & $d_{j i}$ & $\theta_{j i}$ & $r_{j i}$ \\
\hline $1_{1}$ & 0 & 1 & 1 & 0 & 0 & 0 & 0 & 0 & $q_{11}$ \\
\hline $1_{2}$ & 0 & 1 & 1 & $\pi / 2$ & $a$ & $\pi / 2$ & 0 & 0 & $q_{12}-a$ \\
\hline $1_{3}$ & 0 & 1 & 1 & 0 & $a$ & $-\pi / 2$ & 0 & $-\pi / 2$ & $q_{13}+a$ \\
\hline
\end{tabular}

Table 2 MDH parameters for the frames corresponding to the leg $i$.

\begin{tabular}{|r|r|r|r|r|r|r|r|r|r|}
\hline$j_{i}$ & $a\left(j_{i}\right)$ & $\mu_{j i}$ & $\sigma_{j i}$ & $\gamma_{j i}$ & $b_{j i}$ & $\alpha_{j i}$ & $d_{j i}$ & $\theta_{j i}$ & $r_{j i}$ \\
\hline $2_{i}$ & $1_{i}$ & 1 & 0 & 0 & 0 & $-\pi / 2$ & 0 & $q_{2 i}$ & 0 \\
\hline $3_{i}$ & $2_{i}$ & 1 & 0 & 0 & 0 & $-\pi / 2$ & 0 & $q_{3 i}$ & 0 \\
\hline $4_{i}$ & $3_{i}$ & 1 & 0 & 0 & 0 & 0 & $d_{4}$ & $q_{4 i}$ & 0 \\
\hline $5_{i}$ & $4_{i}$ & 1 & 0 & 0 & 0 & $\pi / 2$ & 0 & $q_{5 i}$ & 0 \\
\hline
\end{tabular}

As proposed in Section 2, the loop constraints can be taken into account by using the robot loop-closure equations [1] and the Lagrange multipliers [4,31]. The loop-closure equations of the Orthoglide can be expressed as [15]

$$
\begin{gathered}
\mathbf{f}=\left[\mathbf{f}_{11}^{T}, \mathbf{f}_{21}^{T}, \mathbf{f}_{12}^{T}, \mathbf{f}_{22}^{T}, \mathbf{f}_{13}^{T}, \mathbf{f}_{23}^{T}\right]^{T}=\mathbf{0} \text { with } \\
\mathbf{f}_{1 i}=\left[\begin{array}{c}
x \\
y \\
z
\end{array}\right]-\mathbf{R}_{0}^{i}\left[\begin{array}{c}
d_{4} \cos q_{2 i} \cos q_{3 i}+a \delta_{i} \\
-d_{4} \sin q_{3 i}+a \delta_{i+1} \\
q_{1 i}-a \bar{\delta}_{i+2}-d_{4} \sin q_{2 i} \cos q_{3 i}+d_{6}
\end{array}\right], \mathbf{f}_{2 i}=\left[\begin{array}{c}
q_{2 i} \\
q_{3 i}
\end{array}\right]+\left[\begin{array}{c}
q_{5 i} \\
q_{4 i}
\end{array}\right], \\
\mathbf{f}_{p}=\left[\begin{array}{c}
x^{2}+y^{2}+\left(z-q_{11}-d_{6}\right)^{2}-d_{4}^{2} \\
\left(x-q_{12}-d_{6}+a\right)^{2}+y^{2}+(z-a)^{2}-d_{4}^{2} \\
x^{2}+\left(y-q_{13}-d_{6}+a\right)^{2}+(z-a)^{2}-d_{4}^{2}
\end{array}\right]=\mathbf{0},
\end{gathered}
$$

where $\mathbf{R}_{0}^{i}$ is the rotation matrix between the leg frame $\left(O_{i}, \mathbf{x}_{1 i}, \mathbf{y}_{1 i}, \mathbf{z}_{1 i}\right)$ and the base frame $\left(O_{0}, \mathbf{x}_{0}, \mathbf{y}_{0}, \mathbf{z}_{0}\right)$ (Fig. $\left.3(\mathrm{c})\right), a, d_{4}$ and $d_{6}$ are geometric parameters 
defined in Fig. 3(b) ( $a=0.34 \mathrm{~m}, d_{4}=0.31 \mathrm{~m}$ and $\left.d_{6}=0.03 \mathrm{~m}\right), \delta_{i}$ is a scalar whose value is equal to 1 if $i=3,0$ if not and $\bar{\delta}_{i}=1\left(\bar{\delta}_{i}=0\right.$, resp.) if $\delta_{i}=0\left(\delta_{i}=1\right.$, resp.).

The matrices $\mathbf{J}_{k}, \mathbf{J}_{t k}, \mathbf{A}_{p}, \mathbf{B}_{p}$ and all positions, velocities and accelerations that are necessary for computing the dynamic model of the real robot can be calculated from (40) and (41) using expressions of Section 2 in a straightforward way.

\subsection{Identification Results}

In this part, experimentations are performed and the dynamic model identification is carried out on the Orthoglide. The actuation of each foot is achieved by a rotary $D C$ motor Sanyo Denki linked to a ball-screw. The actuators are powered by current source amplifiers Sanyo Denki. The global drive gains given by the manufacturer are equal to $637 \pi \approx 2001$. Actuator positions and current references are provided by the controller.

The approach presented in Section 3 requires that the robot moves on two types of exciting trajectories:

1. a first type of trajectory without any payload and

2. a second type of trajectory with a payload.

It should be mentioned that those trajectories can be different.

An example of exciting trajectory obtained using the approach recalled in Section 3 is shown in Fig. 4. In our experiments, the payload mass has been measured with an accurate weighing machine $\left(M_{7}=1.983 \mathrm{~kg} \pm 0.001 \mathrm{~kg}\right)$.

Even paying attention to the choice of the exciting trajectories, some small parameters remain poorly identifiable because they have no significant contribution in the joint torques. These parameters have no significant estimations and can be cancelled in order to simplify the dynamic model. Thus parameters for which the relative standard deviation $\% \sigma_{\hat{\chi}_{r i}}$ is too high are cancelled to keep a set of essential parameters of a simplified dynamic model with a good accuracy [26]. The essential parameters are calculated using an iterative procedure starting from the base parameters estimation. At each step the base parameter which has the largest relative standard deviation is cancelled. A new IDIM-TLS parameter estimation of the simplified model is carried out with new relative error standard deviations $\% \sigma_{\hat{\chi}_{r i}}$. The procedure ends when $\max \left(\% \sigma_{\hat{\chi}_{r i}}\right) / \min \left(\% \sigma_{\hat{\chi}_{r i}}\right)<r_{\sigma}$, where $r_{\sigma}$ is a ratio ideally chosen between 10 and 30 depending on the level of perturbation in $\mathbf{Y}$ and $\mathbf{W}$. In the following of the paper, this ratio is fixed to 10 .

Table 3 presents the identification results. Subscript " $R$ " stands for the parameters that have been regrouped using the procedure presented in Section 3. Their 


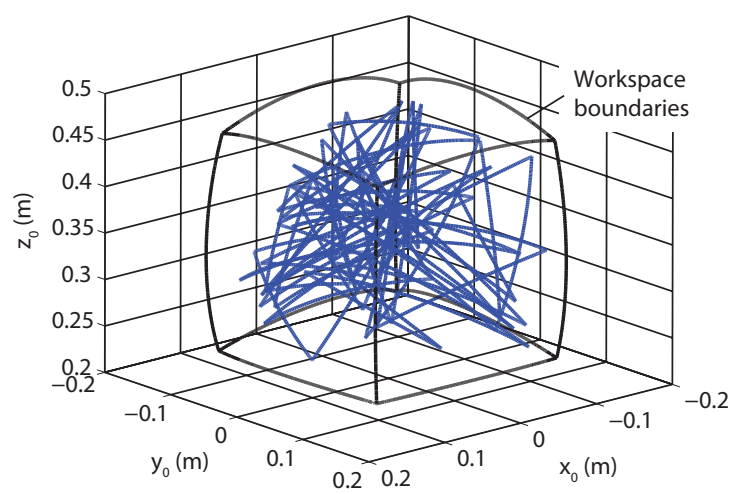

(a) Platform trajectory (duration: 20s)

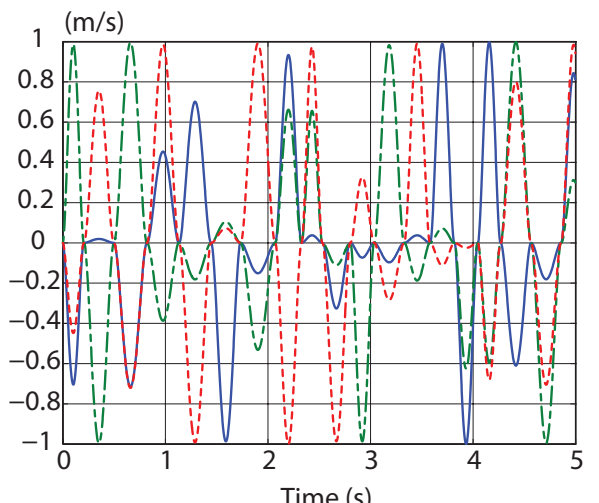

(b) Actuator velocities on the time interval $(0,5 s)$

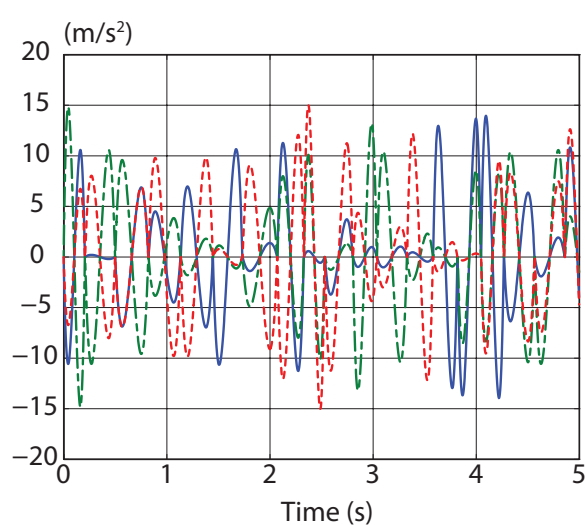

(c) Actuator accelerations on the time interval $(0,5 s)$

Fig. 4 Example of exciting trajectory (blue dotted line: actuator 1; green dash-dotted line: actuator 2 ; red dashed line: actuator 3$)$.

expressions are listed below (for $i=1,2,3$ ):

$$
\begin{aligned}
m_{1_{j} R} & =m_{1_{j}}+i a_{1_{j}}+m_{2_{j}}-3.2258 m x_{3_{j}}+m_{3_{j}}, \text { for } j=1,2 \\
m_{1_{3} R} & =m_{1_{3}}-0.10197 \tau_{o f f_{1_{3}}}+m_{2_{3}}-3.2258 m x_{2_{3}}+m_{2_{3}} \\
i a_{1_{3} R} & =i a_{1_{3}}+0.10197 \tau_{o f f_{1_{3}}} \\
z z_{2_{i} R} & =z z_{2_{i}}+y y_{3_{i}}-0.31 m x_{3_{i}}+y y_{4_{i}} \\
m y_{2_{i} R} & =m y_{2_{i}}+m z_{3_{i}}+m z_{4_{i}} \\
f v_{2_{i} R} & =f v_{2_{i}}+f v_{5_{i}} \\
f s_{2_{i} R} & =f s_{2_{i}}+f s 5_{i} \\
\tau_{o f f_{2_{i} R}} & =\tau_{o f f_{2_{i}}}-\tau_{o f f_{5_{i}}} \\
x x_{3_{i} R} & =x x_{3_{i}}-y y_{3_{i}}+0.31 m x_{3_{i}} \\
z z_{3_{i} R} & =x z_{3_{i}}-0.31 m z_{4_{i}} \\
f v_{3_{i} R} & =f v_{3_{i}}+f v_{4_{i}} \\
f s_{3_{i} R} & =f s_{3_{i}}+f s_{4_{i}} \\
\tau_{o f f_{3_{i} R}} & =\tau_{o f f_{3_{i}}}-\tau_{o f f} f_{4_{i}} \\
m_{6 R} & =m_{6}+\sum_{i=1}^{3} 3.2258 m x_{3_{i}}+m_{4_{i}}+m_{5_{i}}
\end{aligned}
$$


As it can be observed, the same regroupings appears for each robot leg. There is a small difference concerning the regrouped parameter $m_{1 i R}$ : as actuated joint 3 is vertical, contrary to joints 1 and 2 that are horizontal, this joint must support the gravity effects applied on the foot of leg 3 (link $1_{3}$ ). This is not the case for joints 1 and 2 . Thus, this is the reason why parameters $m_{13 R}$ and $i a_{13 R}$ are identified separately.

In Table 3, the given values of standard deviation and percentage of error for the payload mass $m_{7}$ are those estimated by the use of the weighing machine. The results show that the identified drive gains are about $5 \%$ off from those given by the manufacturer.

In Fig. 5, the actuator torques calculated with the relation (17) $\tau=\mathbf{v}_{\tau} \hat{\mathbf{g}}_{\tau}$ (where $\mathbf{v}_{\tau}$ is the measured motor current reference and $\hat{\mathbf{g}}_{\tau}$ the vector of the identified drive gains) are compared with torques computed using the IDIM (21) $\tau_{i d m}=\mathbf{I D M} \hat{\chi}$ (where $\hat{\chi}$ are the identified dynamic parameters). It should be noted here that the trajectory used for plotting these figures is different from the trajectories used in the identification process, i.e. the model has been crossvalidated. It can be seen that the torques are well calculated using the identified IDIM.

In order to definitively validate our method, a second payload of $1.136 \mathrm{~kg}$ is mounted on the platform and a classical IDIM-WLS identification is performed [22]. Two cases are considered:

- (Case 1) the drive gains used are those of the manufacturer and,

- (Case 2) the drive gains are those identified in Table 3.

The following results are found:

- (Case 1) $m_{7}=1.09 \mathrm{~kg} \pm 0.02 \mathrm{~kg} \mathrm{~kg}$,

- (Case 2) $m_{7}=1.14 \mathrm{~kg} \pm 0.02 \mathrm{~kg} \mathrm{~kg}$.

It is thus possible to conclude that the identification of the drive gain using IDIM$T L S$ leads to better payload estimation.

Table 3 Essential parameters of the Orthoglide.

\begin{tabular}{|r|r|r||r|r|r||r|r|r|}
\hline Par. & \multicolumn{1}{|c|}{ Val. } & $\% \sigma_{\hat{\chi}_{r i}}$ & Par. & Val. & $\% \sigma_{\hat{\chi}_{r i}}$ & Par. & Val. & $\% \sigma_{\hat{\chi}_{r i}}$ \\
\hline$g_{\tau_{1}}$ & 2170 & 0.39 & $g_{\tau_{2}}$ & 2100 & 0.39 & $g_{\tau_{3}}$ & 2070 & 0.24 \\
\hline$m_{11 R}$ & 9.40 & 0.44 & $m_{12 R}$ & 8.95 & 0.44 & $m_{13 R}$ & 0.87 & 1.12 \\
\hline$i a_{11 R}$ & - & - & $i a_{12 R}$ & - & - & $i a_{13 R}$ & 8.05 & 0.25 \\
\hline$f v_{11}$ & 85.7 & 0.42 & $f v_{12}$ & 86.7 & 0.50 & $f v_{13}$ & 84.0 & 0.27 \\
\hline$f s_{11}$ & 35.2 & 0.43 & $f s_{12}$ & 41.7 & 0.42 & $f s_{13}$ & 24.1 & 0.32 \\
\hline$m x_{21}$ & -0.12 & 3.75 & $m x_{22}$ & - & - & $m x_{23}$ & - & - \\
\hline$f v_{21 R}$ & 5.57 & 1.36 & $f v_{22 R}$ & - & - & $f v_{23 R}$ & - & - \\
\hline$\tau_{o f f_{21 R}}$ & 0.96 & 1.10 & $\tau_{o f f_{22 R}}$ & - & - & $\tau_{o f f_{23 R}}$ & -0.47 & 2.16 \\
\hline$f v_{31 R}$ & - & - & $f v_{32 R}$ & - & - & $f v_{33 R}$ & -5.57 & 1.46 \\
\hline$f s_{31 R}$ & 1.76 & 1.34 & $f s_{32 R}$ & 1.61 & 1.45 & $f s_{33 R}$ & 1.35 & 1.93 \\
\hline$m_{6 R}$ & 0.92 & 0.86 & $m_{7}$ & 1.98 & 0.05 & & & \\
\hline
\end{tabular}

Finally, to test its effectiveness, the identified dynamic model is used in a model based feedforward control (Computed Torque Control $(C T C)$ ). Tracking errors are compared with those obtained with four different control laws:

1. control of the robot using a simple PID controller, 


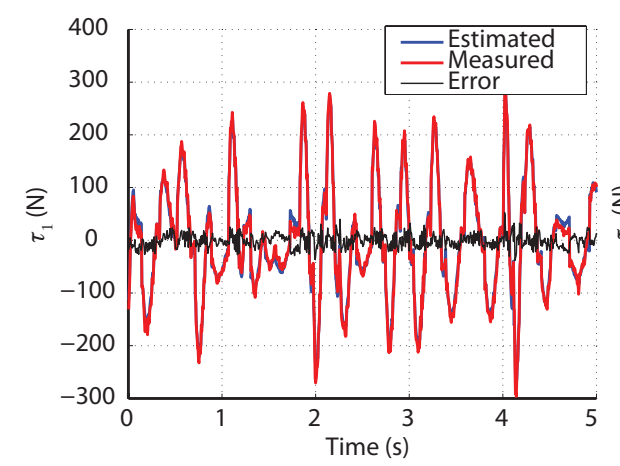

(a) Actuator 1

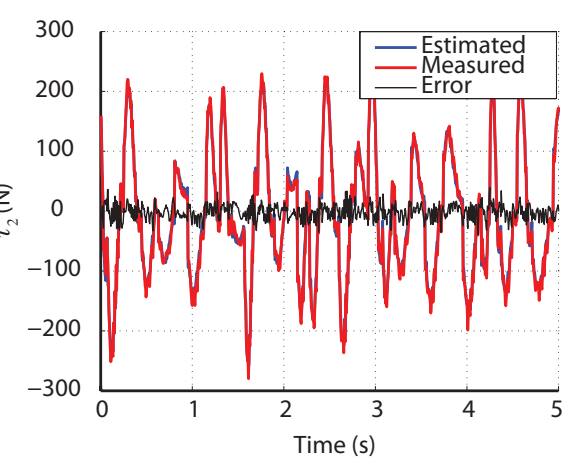

(b) Actuator 2

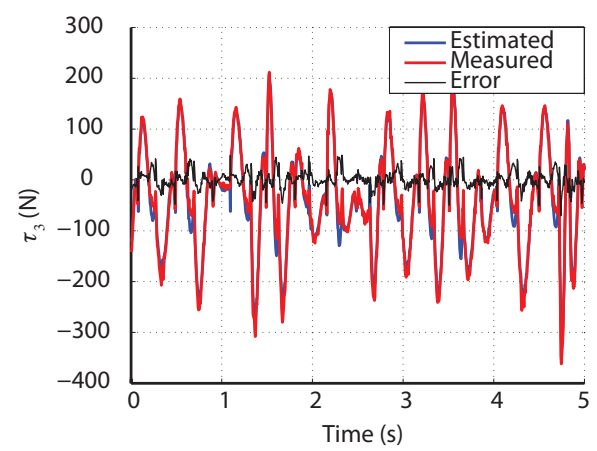

(c) Actuator 3

Fig. 5 Measured and computed torques of the Orthoglide with the payload of $1.983 \mathrm{~kg}$.

2. control of the robot using an advanced PID controller with velocity feedforward,

3. control of the robot using a $C T C$ with a dynamic model identified using the manufacturer's drive gains,

4. control of the robot using a $C T C$ with a dynamic model identified applying $25 \%$ of error on the manufacturer's drive gains

keeping the same bandwidth $(100 \mathrm{rad} / \mathrm{s})$ for the linear part of the five control laws, so that the results can be fairly compared. It should be mentioned that the last case is introduced into the comparison as the identified gains are very close to the manufacturer's gains, so that the effect of drive gains obtained with a larger error can be analyzed.

Two different trajectories are tested. They are plotted in Fig. 6. A mass of 1.983 $\mathrm{kg}$ is fixed on the platform. The nominal value of this mass is directly introduced into the dynamic models used in the $C T C$. The tracking errors in each case for all the three actuated axes are plotted in Figs. 7 and 8. It is obvious that $C T C$ strategies lead to lower tracking errors and are about 40 times better than the simple PID control. However, it is difficult to analyze which $C T C$ strategy is the best. Therefore, in Table 4 are presented the values of the maximal absolute tracking errors and the root-mean-square $(\mathrm{rms})$ errors along the trajectories for each control strategy. Each trajectory is run twice and the presented values are the 
mean values of the obtained results. It can be observed that $C T C$ with identified gains leads to slightly better results, even if the difference with respect to the $C T C$ with manufacturer's gains is not really relevant. This can be explained by the fact that, for $C T C$ strategies with identified and manufacturer's gains, the computed torques are slightly the same, as the difference in the gains is very small (less than $5 \%$ ). It can also be observed that when the gains have an error of $25 \%$, the tracking errors are clearly higher.

Table 4 Tracking errors along the trajectories.

\begin{tabular}{|c|c|c|c|c|c|c|}
\hline Trajectory 1 & \multicolumn{3}{|c|}{ Maximal error $(\mu \mathrm{m})$} & \multicolumn{3}{|c|}{$r m s(\mu \mathrm{m})$} \\
\hline Control strategy & joint 1 & joint 2 & joint 3 & joint 1 & joint 2 & joint 3 \\
\hline Simple $P I D$ & 5165 & 5202 & 335 & 1755 & 1776 & 84 \\
\hline Advanced PID & 453 & 506 & 126 & 158 & 177 & 18 \\
\hline$C T C$ with manuf. gains & 180 & 177 & 96 & 61 & 62 & 10 \\
\hline$C T C$ with identif. gains & 179 & 177 & 96 & 60 & 61 & 10 \\
\hline$C T C$ with error on gains & 188 & 184 & 93 & 66 & 66 & 10 \\
\hline Trajectory 2 & \multicolumn{3}{|c|}{ Maximal error $(\mu \mathrm{m})$} & \multicolumn{3}{|c|}{$r m s(\mu \mathrm{m})$} \\
\hline Control strategy & joint 1 & joint 2 & joint 3 & joint 1 & joint 2 & joint 3 \\
\hline Simple $P I D$ & 9910 & 12610 & 10469 & 5307 & 6371 & 5856 \\
\hline Advanced $P I D$ & 727 & 919 & 842 & 420 & 449 & 429 \\
\hline$C T C$ with manuf. gains & 305 & 339 & 383 & 140 & 149 & 156 \\
\hline$C T C$ with identif. gains & 302 & 339 & 382 & 137 & 147 & 154 \\
\hline$C T C$ with error on gains & 350 & 353 & 409 & 154 & 164 & 169 \\
\hline
\end{tabular}

All these result show the necessity of identifying the drive gains. For doing so, the presented effective approach can be used: for calibrating the drive gains, it is only necessary to weigh the payload mass and to carry out exciting trajectories on the robot.

Finally, to conclude this section and to claim that the proposed method can be applied to any kind of robots (serial or parallel, with any types of dof), we would like to mention that it has been experimentally tested on three 6 dof industrial robots (the Staübli TX-40 (2kg of payload) and RX-90 robots (about 10kg of payload) and the Kuka KR270 robot (270kg of payload)). Experimental results have either confirmed the drive gains values given by the manufacturers or have shown significant improvements of the identification of the drive gains values leading to better payload estimations.

\section{Conclusion}

This paper has presented a new method for the global identification of the parallel robot dynamic parameters including the whole gains of the total drive chain. The presented work has:

- proposed a systematic and straightforward procedure for the computation of the IDIM for parallel robots combined with a way to obtain a set of symmetric base parameters when robot legs are identical,

- shown a new method for the global identification of all robot dynamic parameters, including joint drive gains. 


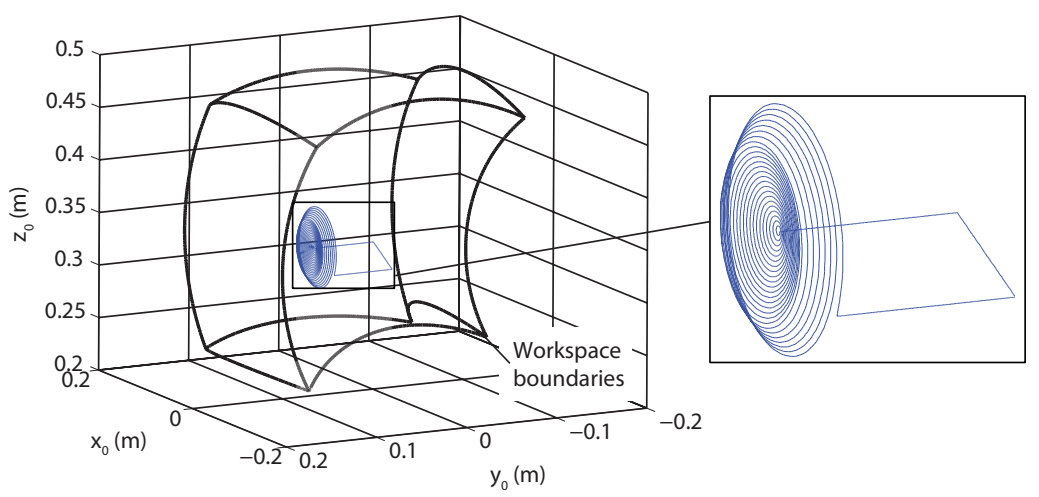

(a) Trajectory 1

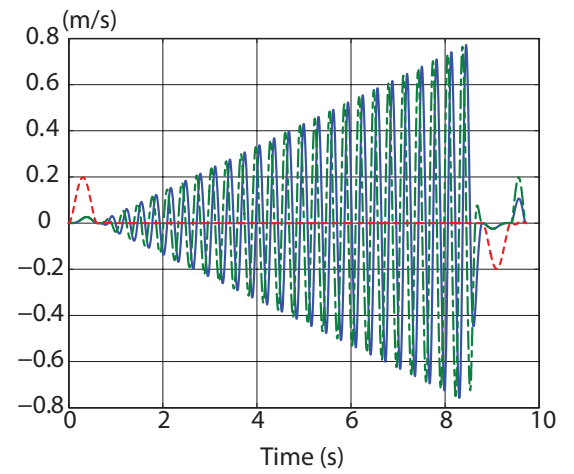

(b) Actuator velocities for trajectory 1

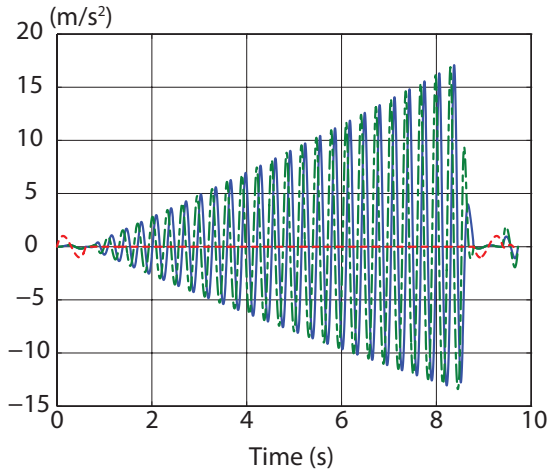

(c) Actuator accelerations for trajectory 1

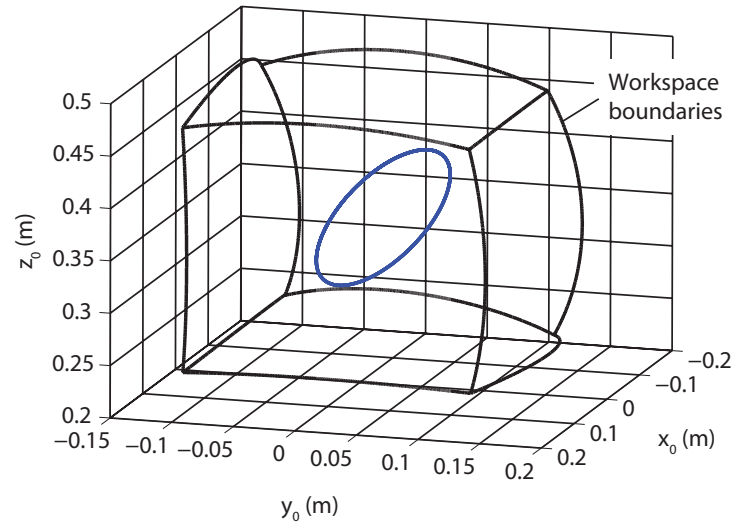

(d) Trajectory 2

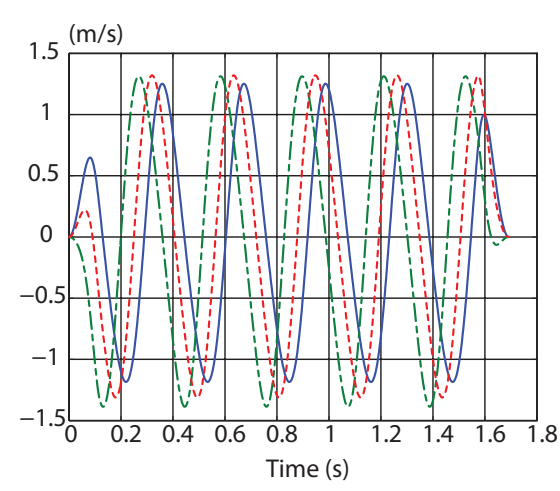

(e) Actuator velocities for trajectory 2

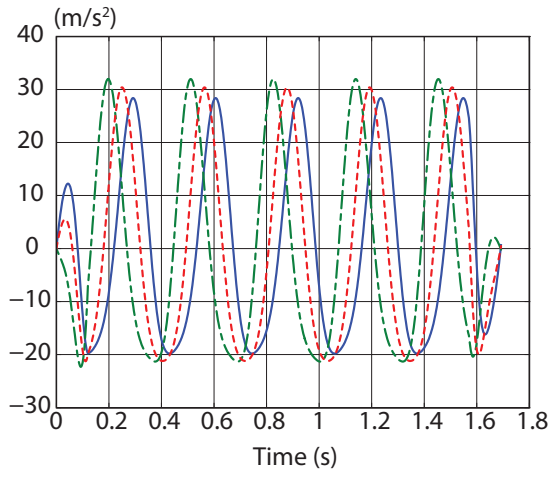

(f) Actuator accelerations for trajectory 2

Fig. 6 The trajectories for testing the control strategies (blue dotted line: actuator 1; green dash-dotted line: actuator 2; red dashed line: actuator 3). 


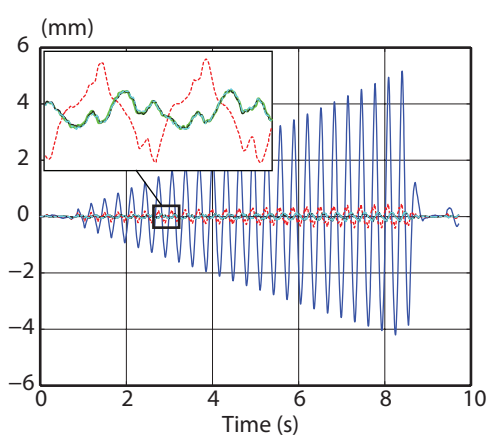

(a) Actuator 1

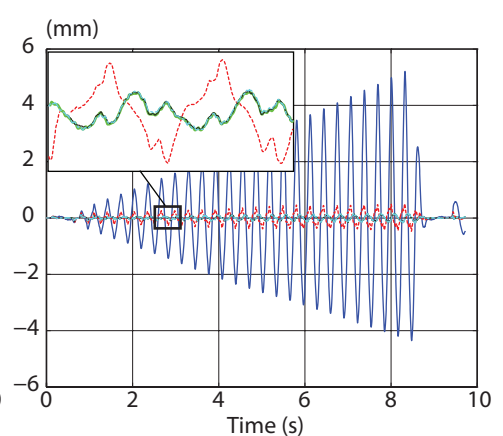

(b) Actuator 2

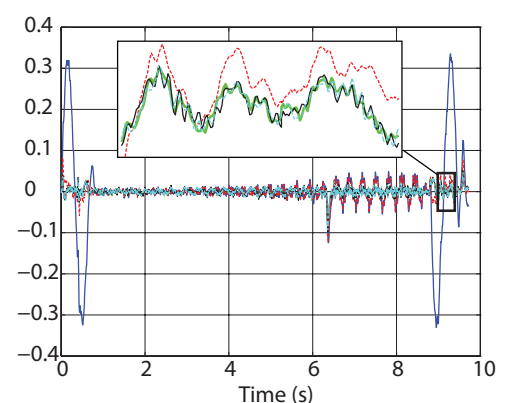

(c) Actuator 3

Fig. 7 Tracking errors for each actuator on trajectory 1 (blue full line: simple PID; red dotted line: advanced PID with velocity feedforward; cyan bold line: $C T C$ with parameters identified using identified drive gains; black thin line: $C T C$ with parameters identified using manufacturer's drive gains; green dash+dotted line: $C T C$ with parameters identified using error on gains).

The identification method is easy to implement and does not need any special test or measurement on the components of the joint drive train. It is based on a IDIM-TLS technique using motor current reference and joint position sampled data while the robot is tracking some reference trajectories without load fixed on the robot and some trajectories with a known payload fixed on the robot endeffector. The ten inertial parameters are measured or calculated by $C A D$ software. The method has been experimentally validated on a prototype of parallel robot with three translational dof, the Orthoglide. The results have shown that the identified gains allows the best payload reconstruction and the best accuracy along a desired trajectory.

Acknowledgements The authors would like to thank Philippe Lemoine for his great help and support during the experimental validations carried out on the Orthoglide in the scope of this work.

\section{References}

1. J.P. Merlet. Parallel Robots. Springer, 2nd edition, 2006. 


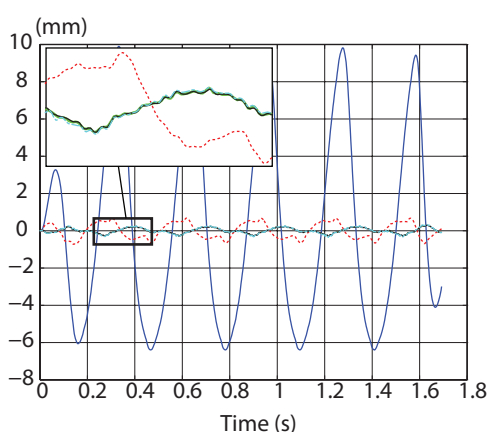

(a) Actuator 1

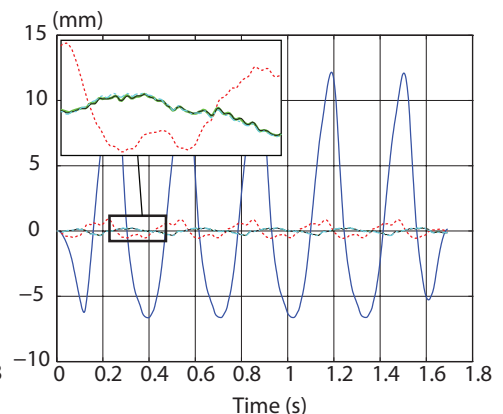

(b) Actuator 2

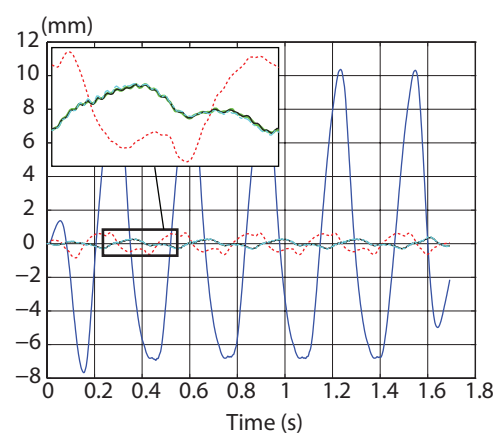

(c) Actuator 3

Fig. 8 Tracking errors for each actuator on trajectory 1 (blue full line: simple $P I D$; red dotted line: advanced PID with velocity feedforward; cyan bold line: $C T C$ with parameters identified using identified drive gains; black thin line: $C T C$ with parameters identified using manufacturer's drive gains; green dash+dotted line: $C T C$ with parameters identified using error on gains).

2. Y. Amiral, G.F. Francois, J. Pontnauand, and M. Dafaoui. Design and control of a new six-dof parallel robot: application to equestrian gait simulation. Mechatronics, 6:227239, 1996.

3. A. Vivas and P. Poignet. Predictive functional control of a parallel robot. Control Engineering Practice, 13:863-874, 2005.

4. W. Khalil and E. Dombre. Modeling, Identification and Control of Robots. Hermes Penton London, 2002.

5. W. Khalil, M. Gautier, and P. Lemoine. Identification of the payload inertial parameters of industrial manipulators. In Proceedings IEEE ICRA, pages 4943-4948, Roma, Italy, April 2007.

6. P.P. Restrepo and M. Gautier. Calibration of drive chain of robot joints. In Proceedings of the 4th IEEE Conference on Control Applications, pages 526-531, 1995.

7. P. Corke. In situ measurement of robot motor electrical constants. Robotica, 23(14):433436, 1996.

8. M. Gautier and S. Briot. New method for global identification of the joint drive gains of robots using a known inertial payload. In Proceedings IEEE ECC CDC, pages 526-531, Orlando, Florida, USA, December 2011.

9. M. Gautier and S. Briot. New method for global identification of the joint drive gains of robots using a known payload mass. In Proceedings IEEE IROS, San Francisco, CA, USA, September 2011.

10. A. Vivas, P. Poignet, F. Marquet, F. Pierrot, and M. Gautier. Experimental dynamic identification of a fully parallel robot. In Proceedings of the 2003 IEEE International Conference on Robotics \& Automation (ICRA 2003), Taipei, Taiwan, 2003. 
11. S. Guégan, W. Khalil, and P. Lemoine. Identification of the dynamic parameters of the orthoglide. In Proceedings IEEE ICRA, pages 3272-3277, Taipei, Taiwan, September 2003.

12. P. Renaud, A. Vivas, N. Andreff, P. Poignet, P. Martinet, F. Pierrot, and O. Company. Kinematic and dynamic identification of parallel mechanisms. Control Engineering Practice, 14:1099-1109, 2006.

13. M. Grotjahn, B. Heiman, and H. Abdellatif. Identification of friction and rigid-body dynamics of parallel kinematic structures for model-based control. Multibody System Dynamics, 11:273294, 2004.

14. M. Diaz-Rodriguez, V. Mata, A. Valera, and A. Page. A methodology for dynamic parameters identification of 3-dof parallel robots in terms of relevant parameters. Mechanism and Machine Theory, 45:13371356, 2010.

15. D. Chablat and P. Wenger. Architecture optimization of a 3-dof parallel mechanism for machining applications, the Orthoglide. IEEE Transactions on Robotics and Automation, 19(3):403-410, 2003.

16. S. Briot and M. Gautier. Global identification of drive gains and dynamic parameters of parallel robots - part 1: Theory. In Proceedings of the 19th CISM-IFToMM Symposium on Robot Design, Dynamics, and Control (RoManSy), 2012.

17. S. Briot and M. Gautier. Global identification of drive gains and dynamic parameters of parallel robots - part 2: Case study. In Proceedings of the 19th CISM-IFToMM Symposium on Robot Design, Dynamics, and Control (RoManSy), 2012.

18. O. Ibrahim and W. Khalil. Inverse and direct dynamic models of hybrid robots. Mechanism and Machine Theory, 45:627-640, 2010.

19. M. Pfurner and M.L. Husty. New Trends in Mechanism Science, chapter Implementation of a new and efficient algorithm for the inverse kinematics of serial 6R chains, pages 91-98. Springer, 2010.

20. S. Briot and V. Arakelian. Optimal force generation of parallel manipulators for passing through the singular positions. International Journal of Robotics Research, 27(8):967-983, 2008.

21. D. N. Nenchev, S. Bhattacharya, and M. Uchiyama. Dynamic analysis of parallel manipulators under the singularity-consistent parameterization. Robotica, 15(4):375-384, 1997.

22. M. Gautier. Dynamic identification of robots with power model. In Proceedings IEEE ICRA, pages 1922-1927, Albuquerque, USA, April 1997.

23. M. Gautier. Numerical calculation of the base inertial parameters. Journal of Robotics Systems, 8(4):485-506, 1991.

24. M. Gautier and W. Khalil. Exciting trajectories for the identification of the inertial parameters of robots. International Journal of Robotics Research, 11(4):362-375, 1992.

25. J. Swevers, C. Ganseman, D. Tukel, J. DeSchutter, and H. VanBrussel. Optimal robot excitation and identification. IEEE Transactions on Robotics and Automation, 13:730740, 1997.

26. W. Khalil and O. Ibrahim. General solution for the dynamic modeling of parallel robots. Journal of Intelligent and Robotic Systems, 49(1):19-37, 2007.

27. S. Van Huffel and J. Vandewalle. The Total Least Squares Problem: Computational Aspects and Analysis. Frontiers in Applied Mathematics series 9. Philadelphia, Pennsylvania: SIAM, 1991.

28. M. Gautier, P. Vandanjon, and C. Presse. Identification of inertial and drive gain parameters of robots. In Proceedings IEEE CDC, pages 3764-3769, Lake Buena Vista, FL, USA, 1994.

29. G.H. Golub and C.F. Van Loan. Matrix computation. J. Hopkins 2nd Ed.

30. M. Gautier and S. Briot. Global identification of drive gains parameters of robots using a known payload. In Proceedings of the 2012 International Conference on Robotics and Automation (ICRA 2012), 2012.

31. F.C Moon. Applied dynamics. J. Wiley and Sons, 2007. 